\title{
Intelligent Collaborative Quality Assurance System for Wind Turbine Supply Chain Management Intelligent Collaborative Quality Assurance System for Wind Turbine Supply Chain Management
}

\author{
B. L. SONG, W.LIAO, J. LEE \\ National Science Foundation (NSF) Center for Intelligent Maintenance System (IMS) \\ Cincinnati, US
}

\begin{abstract}
To determine the root causes or sources of variance of bad quality in supply chains is usually more difficult because multiple parties are involved in the current global manufacturing environment. Each component within a supply chain tends to focus on its own responsibilities and ignores possibilities for interconnectivity and therefore the potential for systematic quality assurance and quality tracing. Rather than concentrating on assigning responsibility for "recall" incidents, it would be better to expend that energy on constructing a collaborative system to assure product quality by employing a systematic view for the entire supply chain. This paper presents a systematic framework for intelligent collaborative quality assurance throughout an entire supply chain based on an expert system for implementing two levels of quality assurance: system level and component level. This proposed system provides intelligent functions for quality prediction, pattern recognition and data mining. A case study for wind turbines is given to demonstrate this approach. The results show that such a system can assure product quality improved in a continuous process.
\end{abstract}

Keywords-Wind turbine expert system; Supply chain management; Collaborative quality assurance; Prediction; Pattern recognition;

\section{INTRODUCTION}

Quality is a critical requirement for customers, especially in the case of expensive and complex products. In recent years, an increasing number of product recalls are occurring. Such product recall incidents have resulted in serious customer dissatisfaction and significant company losses in both image and business. Rather than argue who ought to apologize to the "recall" incidents, it would be better to expend that energy on constructing a collaborative system to assure product quality by employing a systematic view for the entire supply chain. A product supply chain can encompass multiple, diverse parties in the current global manufacturing environment. Each functional part in supply chain tends to focus on its own responsibilities, resulting in a lack of strong interconnected infrastructure to support systematic quality assurance, such as clear management structure and a quality tracing enabled data collection framework. It is therefore often difficult to identify or trace the exact reason for bad quality. How to ensure product quality collaboratively becomes a vital task for the companies along the supply chain.
Supply chain management (SCM) was defined as the management of a network of interconnected businesses involved in the ultimate provision of product and service packages required by the end customers. It spans all movement and storage of raw materials, WIP inventory and finished goods from point-of-origin to point-of-consumption. A good SCM is essential for companies to meet global competition. Nowadays, many manufacturers and service providers collaborate with their suppliers and upgrade their purchasing and supply management functions from a clerical role, to an integral part of SCM. In terms of possessing a systematic quality assurance function and a collaborative data collection framework for quality tracing, gaps still exist.

Quality assurance was defined as "a strategic management function concerned with the establishment of policies, standards and systems for the maintenance of quality"[1]. Later, as a result of benchmarking studies, Baines and Ryan determined that quality assurance could be identified as [2]:

1) A tool to demonstrate regulatory compliance;

2) A business efficiency tool to ensure product quality and minimize hygienic risks;

3) A communication tool to customers and consumers, wherever they are in the world.

Quality assurance becomes increasingly important in integrated SCM [3-4]. As for the methodologies, the majority of prior works tend to conduct failure testing or statistical control to improve quality assurance system [5-9]. Over the past thirty years, considerable advances have been made in computational intelligence. Various intelligent technologies and/or algorithms like artificial neural networks genetic algorithms, fuzzy/logic systems, learning algorithms, and metaheuristics have been developed for realizing intelligent control or expert systems [10]. Compared to traditional statistical control based quality assurance, computational intelligence technologies have advantages for making intelligent decisions such as quality prediction and pattern recognition for the situations with high complexity. If the quality can be predicted before production, it will greatly help avoid generating bad products. Designer can forecast the quality and thus optimize designed settings and tighter tolerance before releasing the design, while downstream parties, such as manufacturing, can optimize real settings in 
the similar way prior to production. And moreover, if the quality can be classified into several levels such as "good quality", "average quality" and "bad quality," products classified lower than "good quality" can be analyzed and traced back to the settings of the impact factors in order to determine the root cause of quality deficiencies. Through such analysis, the rules of best settings can be obtained, and the necessary changes in product or process design can be determined. Hence, providing intelligent functions such as quality prediction, pattern classification and data mining can improve quality by improving overall design; in addition, such functions can help optimize quality assurance for other downstream parties as well. Currently, there exists little research [11] on developing such intelligent systems for quality improvement in industry process. Extensive space could be explored to improve the performance of collaborative quality assurance.

With the consideration of the significance in closing above mentioned gaps, this paper will base on computational intelligence technologies to establish a collaborative quality assurance expert system for machinery products to ensure and improve their quality continuously.

This paper is structured as follows. In Section 2, the methodology of collaborative quality assurance in SCM is proposed. Section 3 takes wind turbine as a case to demonstrate the methodology. Finally, conclusions and future work are provided in Section 4.

\section{Methodology For Collaborative QuAlity ASSURANCE IN SCM}

Methodology to develop the collaborative quality assurance expert system in SCM is provided in this section. Quality and quality assurance will be defined first. Three conceptual frameworks, including a management model, a technical model and a database management model, will be established to help guide the coordination of quality assurance along the whole supply chain. Intelligent functions such as quality prediction, pattern recognition and knowledge mining will be designed in the technical model to support two levels of collaborative quality assurance in SCM: system level and component level.

\section{A. Problem formulation}

1) Quality

It is important to realize that quality is determined by the intended users, clients or customers, not by society in general. 'Expensive' does not always mean 'high quality'. Even goods with low prices can be considered quality items if they meet a market's requirements.

Quality ultimately is measured in terms of customer satisfaction. Customers may have various measurements, such as number of product recalls, number of maintenance fix requests per year, defects found after product delivery per function point, cost of defects (e.g. annual maintenance costs), costs of quality activities (e.g. costs of inspections, diagnostics, test execution, defect tracking, preventive measures and QA education), mean time between failure (MTBF), mean time to repair (MTTR), and so on, to check the product quality characteristics like functionality, reliability, safety, efficiency, and maintainability.

For this paper, no matter what a customer's requirements are, a product that is closest to meeting these requirements is considered to be of good quality.

\section{2) Quality Assurance}

Quality Assurance, or QA for short, was described as a set of activities intended to ensure that products (goods and/or services) satisfy customer requirements in a systematic, reliable fashion. QA can't absolutely guarantee the production of quality products, unfortunately, but makes this more likely. Two objectives are therefore set for achieving the optimal QA (see Figure 1).

\section{a) Objective 1: To optimize the design of product}

Customer requirements will be translated by designers into parameters for what constitutes a high quality product. This paper assumes that designers will provide the optimal value setting and tolerance of all possible quality impact factors in advance, including raw material specification, product dimensions, manufacturing environment, machine statuses, delivery requirements and so on. The evaluation criterion of an optimal design is that the design can best meet customerdefined requirements. The objective 1 is identified as:

To Minimize Difference 1 = Design Setting - Customer Requirement

\section{b) Objective 2: To maximize the compliance with design}

Given an optimal design, quality assurance will attempt to make the real value setting of each quality impact factor along supply chain (e.g. raw material, manufacturing, delivery) in line with those of the design, so as to meet the functionality or the specifications of design. This objective can also be represented as minimizing the difference between real settings and design settings, that is:

\section{To Minimize Difference 2 = Real Setting - Design Setting}

The difference 2 is essentially the deviation of real settings from design settings. A maximum acceptable deviation from a nominal design setting is called tolerance.

The scales of the differences of different factors are not the same. A Compliance Index (CI) is defined to represent the degree of accuracy of the real setting in comparison to the design setting in a standardized scale from 0 to 1 . A value of 0 means that the real setting matches design requirements perfectly, while a value of 1 means that real settings do not adequately match design requirements. These values can be obtained by

$$
\begin{aligned}
& \boldsymbol{C I}=1-e^{-\left|\frac{R-D}{D}\right|} \\
& \text { s.t. } \quad \boldsymbol{R}>=0, \boldsymbol{D}>0
\end{aligned}
$$

where $\mathrm{R}$ and $\mathrm{D}$ represent the real setting and design setting for a same impact factor, respectively. If the setting of certain input factors for the best design is zero, then the following 
equation could be used to calculate the compliance index:

$$
\begin{aligned}
& \boldsymbol{C I}=1-\boldsymbol{e}^{-|\boldsymbol{R}-\boldsymbol{D}|} \\
& \text { s.t. } \quad \boldsymbol{R}>=0, \boldsymbol{D}>=0
\end{aligned}
$$

Objective 2 is therefore to "Minimize CI".

Accordingly, QA includes two activities in this study: to design the best regulation of the quality related factors which rely on raw materials, assembly, components and overall products, as well as the services related to production, inspection and delivery processes; and to seek the maximum compliance between each real factor setting and their corresponding design factor settings.

\section{3) Closed loop quality assurance improvement to guarantee good}

An expert system based closed loop quality assurance improvement process is proposed in Figure 1 to fulfill the above two objectives continuously. There are multiple factors (e.g. raw material factors, manufacturing environment factors, machine factors, assembly factors, delivery factors, etc) throughout supply chain that may influence product quality in various ways and in dynamic situations. The individual and combined influence of multiple factors on quality is unclear. The rules of knowledge base will be obtained from the intensive study of the impact of multiple factors on product quality by the expert system. The product quality will be predicted and patterns will be recognized. The features indicative of bad quality will be extracted and feedback to designers to determine, and improve upon, parameter settings and tolerances for bad quality. The deviation of real settings from nominal design settings on multiple factors will also be studied in order to provide knowledge for compliance improvement. Once the root causes for bad quality are determined, they will be delivered to appropriate personnel for quality improvement. The above improvement of design and compliance will be made in a continuous process.

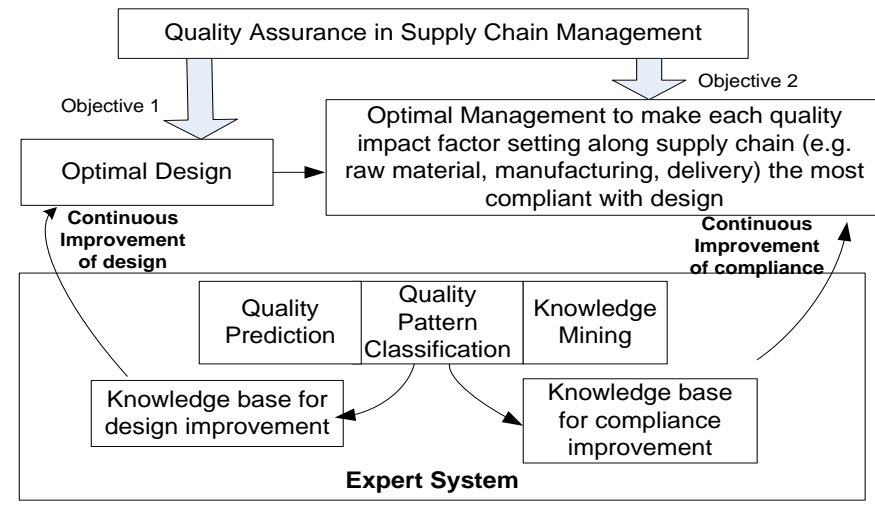

Fig. 1. Closed Loop Improvement Method To Guarantee A Good Quality Assurance In SCM.

The management-level method for guaranteeing the quality impact factor setting's compliance with design is demonstrated in Section 2.2, while the expert system for studying multi-factor impacts on quality, providing intelligent functions such as prediction, pattern recognition, and generating the knowledge bases for above two objectives is presented in Section 2.3. The distributed database management system proposed in Section 2.4 can help a user trace back the situation in which a quality issue has been discovered.

\section{B. Management Structure for collaborative quality assurance}

Assuming that a certain design is optimal, an order oriented collaborative quality assurance team management mode is proposed in order to assure the supply chain factor settings' compliance with the design.

For the products of the same order, a quality assurance (QA) team will be organized among all the parties along the supply chain. The system supplier needs to take direct responsibility for delivering the product (a complete assembled system is defined as a product) to end users and it also needs to guarantee the quality of the components outsourced. The system supplier has a good connection to the end user and component suppliers. Considering these connections, the system supplier is the best party to lead the quality assurance team. Each quality related department, such as system level design, assembly process and delivery, component level design, raw material supply, manufacturing, and delivery will work together for QA.

After receiving the customer expectations of what they expect from a product, system level QA will design the optimal regulations (e.g. parameter value setting and tolerance setting for materials, manufacturing and delivery along supply chain) for the best quality product. The quality parameters for components will be delivered to each component QA as a customer requirement. Component QA will continue to update the optimal settings for a component if the customer requirements received need to be amended, or if new requirements are identified. The designed settings will then be passed to each party along the component supply chain.

Each downstream party will check the compliance of real settings with design settings after it completes its part. If the completed part passes the quality compliance check, then it will be delivered to its upstream party. If all parties, including those responsible for raw materials, manufacturing and delivery, have finished their check, a total quality assurance (TQA) will be carried out before the completed component is sent for system level assembly. System level QA will start after all component level QAs are completed. The same quality compliance check will be conducted for the system assembly process and its delivery path. The customer will do a TQA for the final assembled product. Those products which fail the TQA will be sent back for rework.

\section{Two levels of quality assurance expert system in SCM}

Ensuring a high level of quality for products throughout a supply chain is a much-needed perspective into the SCM due to the importance of quality in terms of improving system reliability and customer satisfaction. Various factors along a supply chain can influence the quality of a product. Determining the impact of multiple factors on product quality is of significant importance in providing useful information to improve quality assurance. Few quality assurance approaches can satisfy this need due to the complexity of the real situation. This section will propose a quality assurance expert system to close this gap. As the product (or system) and each 
of its components have their own supply chains, the framework for the expert system is developed based on two levels: system-level quality assurance and component-level quality assurance.

\section{1) System level quality assurance in SCM}

The procedure for conducting system level quality assurance is illustrated in Figure 2. As indicated in the procedure, the supply chain of the product (or system) is identified first. The criteria for what constitutes system-level quality, such as reliability, based on the customer requirements, are then defined. Component level quality assurance is one important step for guaranteeing system level quality assurance. Theoretically speaking, quality assurance for each component will ensure the optimal result, in terms of quality, for the overall product. However in reality, it is not necessary to research each component, and it would be costly to do so. Thus, it becomes essential to balance the costs versus the benefit first. For the situation in which it is not cost effective to do quality assurance for each component, the critical component analysis and selection needs to be conducted. Subsequently, it is vital to define component-level quality for the chosen critical component(s) in order to conduct component-level quality assurance. The factors of the component that are most indicative of quality will be selected for studying their impact on the defined component-level quality. With this information, the component-level quality assurance expert system can be constructed. The comparison of component quality performance before and after applying the proposed system will be used for evaluation and to determine if improvements to the system can be made. The customer's requirements are another criterion for making a quality determination. The order of using these two criteria can be adjusted according to the target of the quality improvement. Once the quality of all the studied components is improved to meet the defined requirements, the overall quality of the product will be evaluated to see whether it can meet the system requirements. If not, the prior steps should be revisited in order to continue improving the component-level quality. Therefore, through the procedure of system-level and component-level quality assurance, the quality of product can be better ensured.

\section{2) Component level quality assurance in SCM}

A component-level quality assurance expert system has been designed (Figure 3) for improving component quality based on the study of the impact of multiple factors on quality. For each component, the selection of factors from different parties along component supply chain, and the quality of the components produced, will be determined. The database system for data collection is presented in following section. Some samples will be selected from the database and input as training data for the expert system.

One major function of the expert system is to provide a scientific way for quality prediction and quality classification. For this purpose, quality forecasting and quality classification models will be built based on the training data. Inputting any test sample of impact factors, the forecasting model will predict the corresponding quality. For the predicted quality, the quality pattern recognition model will identify the pattern that it belongs to. The designer can use this system to test if the design settings will result in a good or bad quality product before releasing the design to downstream parties. Downstream parties, such as manufacturing, can apply the proposed system to predict the quality of products to be produced based on simulated real settings. They are then able to optimize their real settings before production. With this intelligent measurement system, tremendous loss from incorrect design settings or incorrect real settings will be saved.

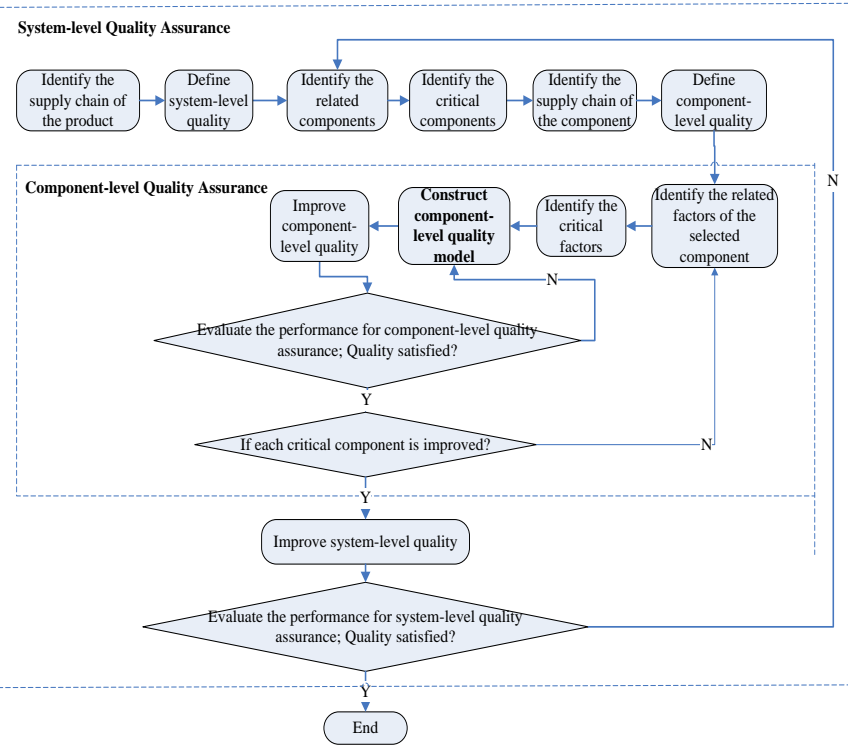

Fig. 2. System level quality assurance in SCM.

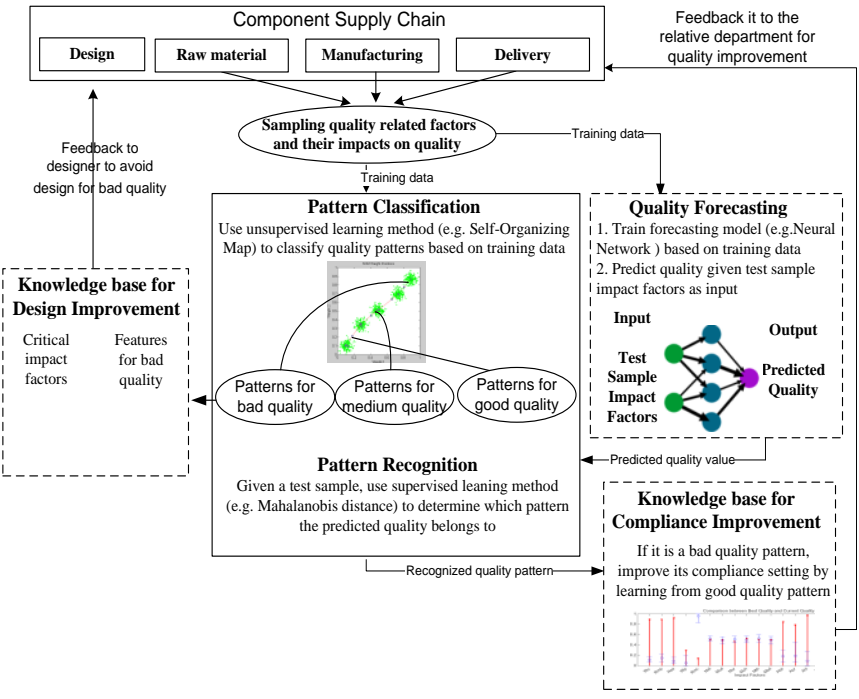

Fig. 3. Component level quality assurance in SCM

The expert system has another function: to generate knowledge bases for design improvement and compliance improvement for achieving the best quality assurance. For instance, a correlation study of the impact factors and their affect on quality will reveal the critical impact factors that have bigger influence on bad quality. Further data mining conducted on bad quality patterns, such as a histogram of each critical impact factor, is helpful to find the features (i.e. range of settings) that result in bad quality. The knowledge obtained 
will be shared with the designer for better design settings and tighter tolerances and hence avoid designs that result in bad quality. For other parties in a supply chain, the analysis of the difference between their input factors and those indicative of good quality will show them how to adjust their settings to improve their compliance with the design settings.

\section{3) Distributed database management system for quality assurance}

Data collection for quality assurance is a challenge because it requires the cooperation of the different parties within the whole supply chain. A good organization with clearly assumed responsibility is important for successful data collection.

This paper thus presented a distributed quality assurance database management system (see Figure 4), in which one quality assurance database (QADB) is associated with each supply chain party, all quality assurance databases are connected via internet, and are under the control of a central database management system (CDBMS). It is suggested that the CDBMS be managed by the system supplier, while the end user has authorization to access and manage the QA information of the products they ordered. Consistent data recording and assessment and documentation integrity is necessary. Each produced part, including components and final product, is attached with an e-tag, such as a Radio Frequency Identification (RFID), which stores its basic information and path for accessing its QADB.

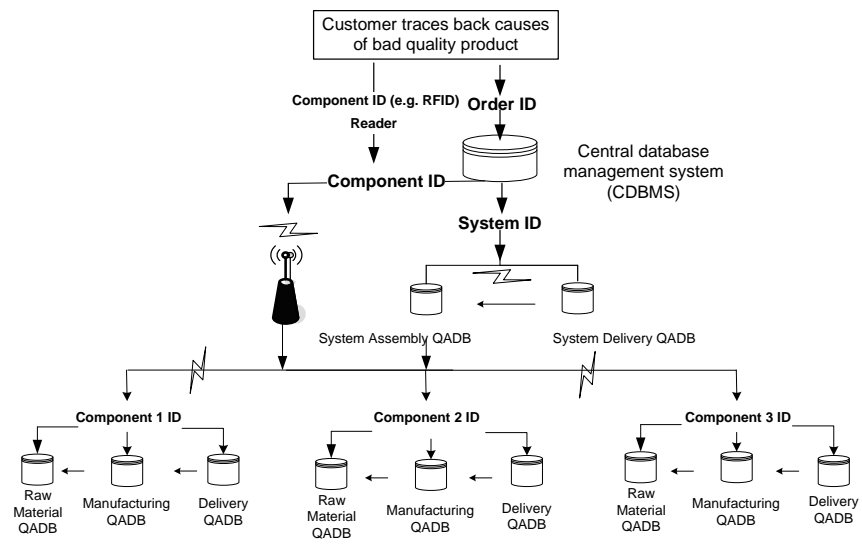

Fig. 4. Distributed quality assurance database management system.

When a quality problem occurs during usage, the end user may trace back its causes. If the source of the problem cannot be located, they may input the order number into the CDBMS to find the system ID and then trace the system delivery status and production status. If there is no problem with the system level process, then the user can continue to trace the issue back to the delivery status, production status and even to the raw material status for each component, with the assistance of each QADB. This process is conducted until it is determined at which stage the quality issue originated.

If the user knows the problem component, he can trace back the component status in the corresponding QADB directly with the assistance of the component ID and other relative information read from its e-tag.

Although manufacturers and suppliers have noticed the importance of quality assurance for wind turbines, they usually confine their quality assurance efforts to their specific realm, which does not make use of potential opportunities for collaboration. For example, designers only focus on how to improve product design, while manufacturers only focus on how to ensure quality during assembly and production and distributers on storage, delivery and logistics. Ignoring the interrelationship between them may easily cause bad products due to poor quality coordination. Thus, it becomes essential to study the relationship between all parts in supply chain and build a collaborative model for quality assurance.

Considering the marvelous long term growth of wind turbine, and its utmost needs for collaborative quality assurance, it will be taken as an example to demonstrate the proposed systematic framework for quality assurance in supply chain based on the study of the relationship between the critical impact factors and product quality. Through the successful application of this proposed methodology, this collaborative quality assurance model can be certainly applicable to some other machinery products.

\section{Identify the supply chain for a wind turbine}

A wind turbine has a unique and internationally distributed supply chain the parameters of which is strongly influenced by the recent increase in wind turbine production. The components of wind turbines have their corresponding supply chains as well. A typical supply chain for a wind turbine is demonstrated in Figure 5. A general supply chain may have warehouses, distribution centers or retailers between the manufacturer and customer. However, in this study, the supply chain can be simplified as a wind turbine is the kind of customized products which is usually directly delivered from a wind turbine manufacturer to their customers.

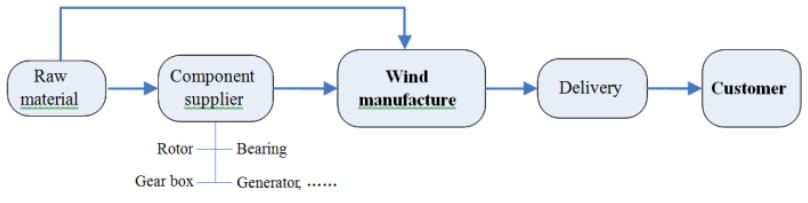

Fig. 5. A typical supply chain for wind turbine.

Based on the framework in Section 2.3, first, system level quality for awind turbine can be defined as high reliability of wind turbine to ensure smooth operation. Through understanding the structure of a wind turbine, its components can be analyzed so as to define component level quality. A collaborative quality assurance model for component level will be discussed in the following sections, which can be applied as quality assurance model for the system level too by defining the system quality index and its corresponding impact factors.

\section{E. Select critical components of wind turbine}

The general structure of wind turbine is shown in Figure 6 [12]. The main parts of a wind turbine (as shown below ) are the rotor, blades, brake, controller, gear box, generator, highspeed shaft, low-speed shaft, nacelle, pitch, wind direction, 
wind vane, yaw drive and yaw motor. Most turbines have either two or three blades. Wind blowing over the blades causes the blades to "lift" and rotate. Generally, the blades and the hub together are called the rotor. When the blades rotate, the gears rotate; the gears connect the low-speed shaft to the high-speed shaft and increase the rotational speeds from approximately 30 to 60 rotations per minute (rpm) to approximately 1000 to $1800 \mathrm{rpm}$, which is the rotational speed required by most generators to produce electricity. Generally, the gear box is the most costly part of a wind turbine.

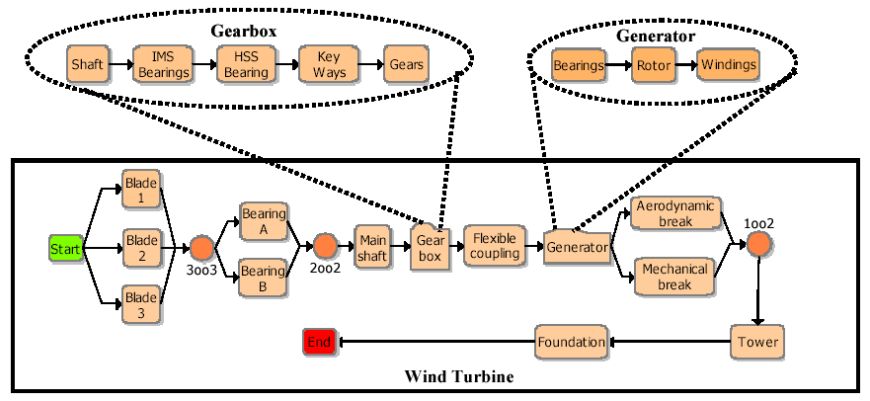

Fig. 6. General structure of wind turbine.

Although a wind turbine is comprised of many components, it is more cost effective to focus on the critical components for quality assurance. In order to identify the critical components of a wind turbine, the typical way is to determine which component has the greatest influence on wind turbine breakdowns. In recent years, numerous failure surveys have been conducted on wind turbines. A downtime distribution was presented in [13], based on a population of German wind turbines. Braam and Tavner both provided recent studies on failure probabilities [14-16]. McMillan and Ault then selected four component categories from these multiple sources and illustrated their annual probability of failure on the same chart [17]. They further used this data to study reliability benchmarks [18]. This paper summarizes their researches in Figure 7 to illustrate the effect of both the failure probability and downtime distribution of four components of wind turbines, namely the electronics \& controls, rotor blades, gearbox and generator. The $\mathrm{X}$ value is the downtime distribution data from Winstats Newsletter[13], meanwhile, while the $\mathrm{Y}$ value is the failure probability from the surveys for the same component [14-16]. It should be noted that each component has three samples marked in different colors. The plot area is divided into four quarters, namely quadrants 1 to 4 , based on the high or low values of the axis. All gearbox \& bearing samples, as well as all generator samples and some blades samples, fall in quadrant 4 , which shows that all these components have a high downtime distribution with comparatively a low failure probability. Therefore, gearbox, generator and rotor blades are taken as more critical components.

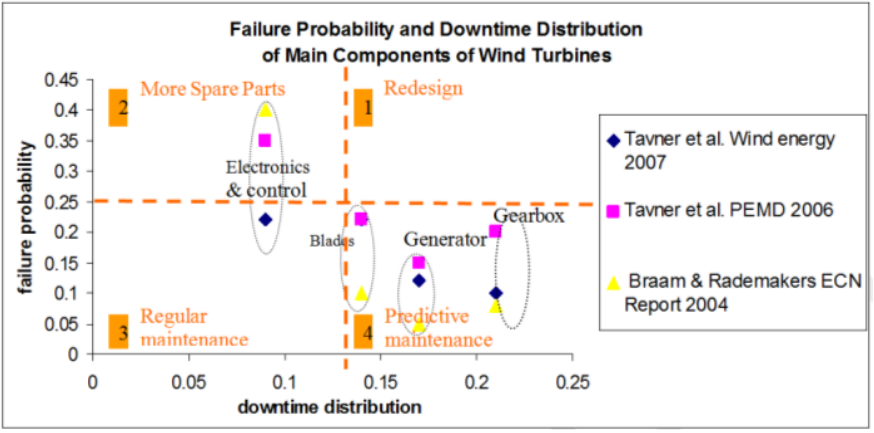

Fig. 7. Wind Turbine Critical Components Selection Based On Integrated Failure Effect Study.

\section{F. Define component level quality}

Component level quality assurance will then be established for the component selected with its corresponding quality criteria (i.e. rules in quality assurance model) and impact factors. As shown in Figure 6, bearings are key subcomponents in both gearboxes and generators, which are two critical components of wind turbines. It is thus taken as an example in the following sections to demonstrate the approach of building component level quality assurance model. This approach can be applied to other components.
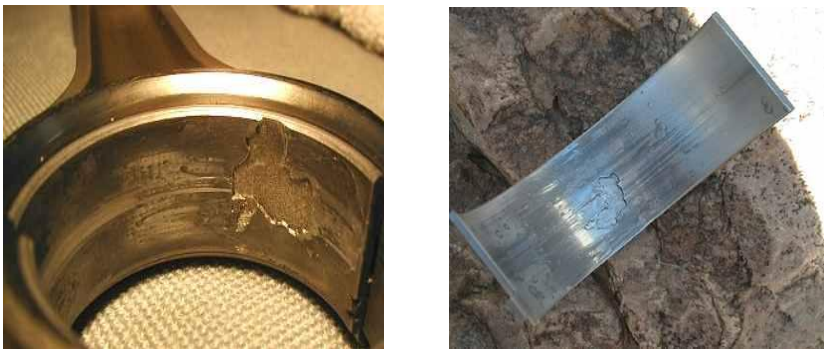

Fig. 8. Fault of bearing.

TABLE I. PRIMARY TYPES OF QUALITY RELATED BEARING DAMAGES.

\begin{tabular}{|c|c|c|c|}
\hline Bearing damage & Caused by & Bearing damage & Caused by \\
\hline \multirow[t]{3}{*}{ wear } & abrasive particles & \multirow[t]{9}{*}{ flaking } & Preloading \\
\hline & inadequate lubrication & & oval compression \\
\hline & vibration & & axial compression \\
\hline \multirow[t]{3}{*}{ smearing } & rollers and raceways & & Misalignment \\
\hline & external surfaces & & Indentations \\
\hline & roller ends guide flanges & & Smearing \\
\hline \multirow[t]{3}{*}{ crack } & rough treatment & & deep seated rust \\
\hline & excessive drive-up & & fretting corrosion \\
\hline & smearing & & Fluting \\
\hline \multirow[t]{4}{*}{ Cage damage } & vibration & \multirow[t]{2}{*}{ corrosion } & deep seated rust \\
\hline & excessive speed & & fretting corrosion \\
\hline & wear & \multirow[t]{2}{*}{ indentation } & Overloading \\
\hline & blockage & & foreign particles \\
\hline
\end{tabular}

The primary types of bearing damages are necessary to be well researched before building the component level quality assurance model. Bearings as prominent parts among the most important components in the majority of machines sometimes, do not always meet their life expectancy due to damages from multiple sources for diverse reasons, such as heavier loading, careless handling, ineffective sealing or unsuitable fits. Each of these factors produces its own particular type of damage and leaves its own special impact on a bearing. Thus, it is 
essential to examine the damaged bearing and study the cause of the damage so as to provide support for quality assurance.

Based on the most prevalent types of bearing damage (see Table 2), it can be seen that most damage is caused by quality issues along the supply chain. Hence, the quality index and the major impact factors influencing bearing quality will be identified and their relationship will be well studied by using this proposed model.

As mentioned before, there exist various types of quality index according to customer requirement. Reliability is adopted in this section to represent the quality of the bearing. Other quality indices can also be considered in the same way, according to a customer's requirements. In engineering, reliability is the ability of a system or component to perform its required functions under stated conditions for a specified period of time. The traditional notation for reliability is $\boldsymbol{R}(\boldsymbol{t})$, while here it is shown as one index of quality, and is denoted as $\boldsymbol{Q}_{r e}$. Mathematically, reliability may be expressed as

$$
Q_{r e}=R(t)=P(T>t)=\int_{t}^{\infty} f(x) d x
$$

where $f(x)$ is the failure probability density function and $t$ is the length of the period of time that is assumed to start from time zero .

\section{G. Identify quality impact factors for critical component}

By analyzing bearing damages and its causes, 19 main quality impact factors which can cover the majority of the causes of damage are selected for study. Five of them are factors related to raw material, six of them are about machining, four of them are machine status factors, three are manufacturing environment factors, and one is the delivery condition. Those 19 impact factors and 1 quality index (i.e. reliability of bearing) are listed in Table 3 .

As stated in the methodology section, the designed settings are assumed to be optimal. The quality assurance target of other parties is to make the real settings meet the designed settings. The deviations of real settings from design settings instead of the real setting values are studied because they can reflect the quality compliance level. The results of which can further help design tighter tolerances. As discussed in Section 2.1, CI is introduced to transfer the different scales of the deviations to a standardized range, from 0 to 1 . In terms of the chosen standard value range, 0 means the difference between real settings and designed settings is the smallest, while 1 means the difference between them is the biggest. $C I\left[R_{l u}\right]$ indicates the compliance index of lubricant in "Raw material" category. Equations (1) and (2) provide the method to calculate $C I$. The $C I s$ of all selected impact factors are taken as the inputs of the quality assurance model. $C I\left[Q_{r e}\right]$, the compliance index of reliability, is the output factor. $C I\left[Q_{r e}\right]$ is calculated by $(1-R(t))$, in order to make it comply with the $C I$ of the input factors. That is 0 means the perfect case with the best reliability, while 1 reflects the worst one.

\section{H. Quality prediction and pattern recognition for critical component}

For the purpose of quality prediction and pattern recognition, many intelligent approaches can be used. In this study, two neural network models, called Feedforward neural network and self-organizing map neural network (SOM) are used. A Feedforward neural network is developed to predict the component quality and a self-organizing map (SOM) is built to classify the quality pattern, through learning the relationship between quality and its impact factors collected along the supply chain.

\section{1) Quality prediction}

A Feedforward neural network is an artificial neural network in which connections between the units do not form a directed cycle.

TABLE II. SELECTED IMPACT FACTORS AND QUALITY INDEX.

\begin{tabular}{|c|l|c|c|}
\hline \multicolumn{2}{|c|}{ Factors } & Notation & $\begin{array}{c}\text { Range } \\
\text { of CI }\end{array}$ \\
\hline Input & & & \\
\hline Raw material & Lubricant & $\mathrm{R}_{\mathrm{lu}}$ & {$[0,1]$} \\
\cline { 2 - 4 } & $\begin{array}{l}\text { mounting } \\
\text { pressure }\end{array}$ & $\mathrm{R}_{\mathrm{mp}}$ & {$[0,1]$} \\
\cline { 2 - 4 } & $\begin{array}{l}\text { seating out of } \\
\text { alignment }\end{array}$ & $\mathrm{R}_{\mathrm{sa}}$ & {$[0,1]$} \\
\cline { 2 - 4 } & foreign particles & $\mathrm{R}_{\mathrm{fp}}$ & {$[0,1]$} \\
\cline { 2 - 4 } & $\begin{array}{l}\text { material } \\
\text { containing }\end{array}$ & $\mathrm{R}_{\mathrm{mc}}$ & {$[0,1]$} \\
\hline \multirow{4}{*}{$\begin{array}{c}\text { Manufacturin } \\
\mathrm{g}\end{array}$} & inner diameter & $\mathrm{M}_{\mathrm{id}}$ & {$[0,1)$} \\
\cline { 2 - 4 } & outer diameter & $\mathrm{M}_{\mathrm{od}}$ & {$[0,1)$} \\
\cline { 2 - 4 } & $\begin{array}{l}\text { surface } \\
\text { roughness }\end{array}$ & $\mathrm{M}_{\mathrm{sr}}$ & {$[0,1)$} \\
\cline { 2 - 4 } & chamfer & $\mathrm{M}_{\mathrm{ch}}$ & {$[0,1)$} \\
\cline { 2 - 4 } & thickness & $\mathrm{M}_{\mathrm{th}}$ & {$[0,1)$} \\
\cline { 2 - 4 } & ball diameter & $\mathrm{M}_{\mathrm{bd}}$ & {$[0,1)$} \\
\hline \multirow{3}{*}{ Machine } & Balance & $\mathrm{A}_{\mathrm{ba}}$ & {$[0,1]$} \\
\cline { 2 - 4 } & Precision & $\mathrm{A}_{\mathrm{pr}}$ & {$[0,1]$} \\
\cline { 2 - 4 } & Health index & $\mathrm{A}_{\mathrm{hi}}$ & {$[0,1]$} \\
\cline { 2 - 4 } & Vibration & $\mathrm{A}_{\mathrm{vi}}$ & {$[0,1]$} \\
\hline \multirow{5}{*}{ Environment } & Temperature & $\mathrm{E}_{\mathrm{te}}$ & {$[0,1]$} \\
\cline { 2 - 4 } & Humidity & $\mathrm{E}_{\mathrm{hu}}$ & {$[0,1]$} \\
\cline { 2 - 4 } & Dust tolerance & $\mathrm{E}_{\mathrm{dt}}$ & {$[0,1]$} \\
\hline \multirow{4}{*}{ Delivery } & $\begin{array}{l}\text { Delivery } \\
\text { condition }\end{array}$ & $\mathrm{D}_{\mathrm{dc}}$ & {$[0,1]$} \\
\hline Output & & & \\
\hline Quality index & Reliability & $\mathrm{Q}_{\mathrm{Re}}$ & $(0,1]$ \\
\hline \multirow{2}{*}{} & & & \\
\hline
\end{tabular}

Note: CI range values have been standardized for all factors.

CI Value range in "Manufacturing" represents the difference between real manufacturing setting and design requirements. 0 means no difference. The closer to 1 , the bigger difference it is. So $0=$ perfect case; $1=$ worst case

It is most commonly used with the back propagation algorithm that often has one or more hidden layers of sigmoid neurons followed by an output layer of linear neurons. In this network, the information moves in only one direction, forward, from the input nodes, through the hidden nodes and to the output nodes. There are no cycles or loops in this type if network. Multiple layers of neurons with nonlinear transfer functions allow the network to learn nonlinear and linear relationships between input and output vectors. A well learned network is often applied for function approximation or regression analysis tasks, including forecasting. Cost function 
is an important concept in learning, as it is a measure of how far away we are from an optimal solution to the problem that we want to solve. Learning algorithms search through the solution space in order to find a function that has the smallest possible cost. A commonly used cost is Mean Square Error (MSE) which tries to minimize the average squared error between the network's output and the target value over all the example pairs.

In this case study, for each selected impact factors and quality index, 1000 samples are collected and then their compliance indices are standardized into a range [0,1]. For each factor, 1000 standardized CI samples are stored in one factor vector, and 1000 quality index samples are in one quality index vector.

In the Feedforward neural network model, the input is the vector of 19 factor vectors, and the output is the quality index vector. Two layers are set and the Levenberg-Marquardt rule is chosen to train this neural network model. The epoch is set to be 1000. The Mean Square Error (MSE) is used to examine the prediction performance. The MSE $<=0.01$ is set as threshold to stop training. After training, Feedforward neural network model could be used to predict $C I$ of quality index (i.e. reliability of bearing) given any test sample.

In Figure 9, the training results of the Feedforward neural network model, including the training state and the corresponding MSE (0.0068) are presented respectively. The results show that this Feedforward neural network model fits the samples to the degree that it is requested, which means this trained Feedforward neural network model can be used to predict the quality index based on the selected impact factors.

The following are two examples in which the proposed model is used to predict the quality index for two test samples:

Test sample vector of impact factors is:

$[0.32576 ; 0.30902 ; 0.30753 ; 0.30937 ; 0.28538 ; 0.43926 ; 0.44336 ; 0.5208$ $1 ; 0.45899 ; 0.53185 ; 0.48846 ; 0.33633 ; 0.3077 ; 0.46696 ; 0.35483 ; 0.0902$ 69;0.044895;0.90836;0.77512].

Quality index is predicted to be: [0.2925]

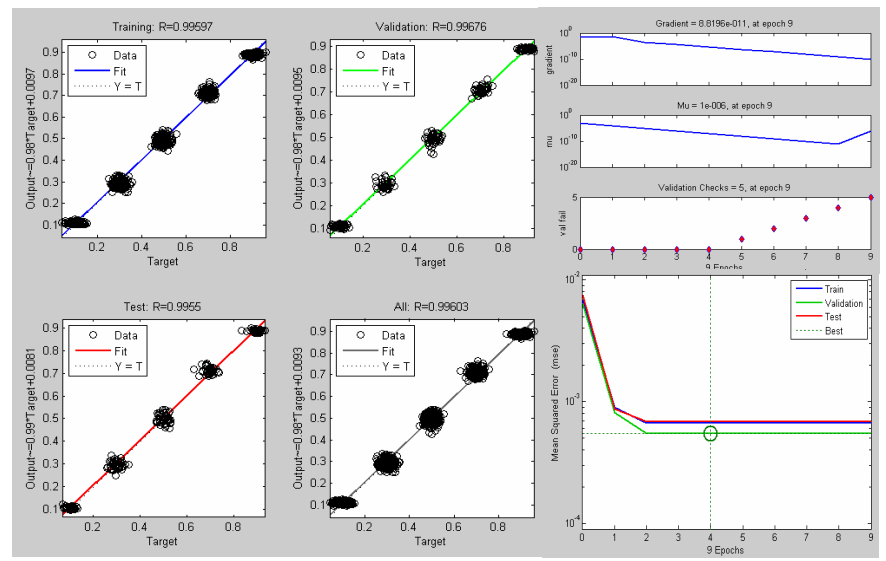

Fig. 9. Training results of Feedforward neural network.
$[0.8927 ; 0.8942 ; 0.9217 ; 0.2920 ; 0.1420 ; 0.5032 ; 0.4932 ; 0.4675 ; 0.5239$; $0.5061 ; 0.5018 ; 0.8447 ; 0.7880 ; 0.9724 ; 0.1794 ; 0.9296 ; 0.9256$; $0.3489 ; 0.3816]$

Quality index is predicted to be: [0.9476]

\section{2) Quality pattern recognition}

In addition to being able to predict a certain quality index value, it would be advantageous to be able to determine the patterns of the whole values as well. For this purpose, SOM has been adopted to classify a certain quality index into various levels. If a certain pattern needs to be deeper studied, a specific analysis can be conducted for that.

SOM is another type of artificial neural network that is trained using unsupervised learning to produce a lowdimensional, discretized representation of the input space of the training samples. It produces a map to represent the input space of the training samples. It is different from other artificial neural networks in the sense that SOM uses a neighborhood function to preserve the topological properties of the input space. SOM has one layer with the neurons organized in a grid, which makes it useful for visualizing lowdimensional views of high-dimensional data, - this function is akin to multidimensional scaling.

SOM operates in two modes like most neural networks: training and mapping. Training builds the map using input samples. Mapping automatically classifies a new input vector. Training is a competitive process, also called vector quantization. Firstly, it will randomize the map's nodes' weight vectors, and then grab an input vector, traverse each node in the map, including the use of Euclidean distance formula to find similarity between the input vector and the map's nodes' weight vector. It also includes the track of the best matching unit (BMU), the node that produces the smallest distance. Afterward, the nodes in the neighborhood of BMU will be updated by pulling them closer to the input vector. The whole procedure will be repeated till current iteration reaches the limit on time iteration.

In this study, 1000 samples of quality index are used as training data to build a SOM neural network for quality pattern classification. Within SOM one layer is set, and the Batch unsupervised weight/bias training algorithm is chosen.

In Figure 10, the results displayed by the developed of SOM are presented, in which 5 classes are obtained, after training. This means that the quality index can be classified into 5 levels: "very good quality", "good quality", "average quality", "bad quality” and "very bad quality”, respectively.

This SOM model can then be used for recognizing the quality pattern given a certain quality index value. For instance, the predicted quality index, 0.2925, in the abovementioned sample test 1 , is classified by the SOM into class 2, which is considered to be of "good quality"; the quality index in sample test 2, 0.9476, is classified by the SOM into class 5, which is considered to be of "very bad quality".

Test sample vector of impact factors is: 


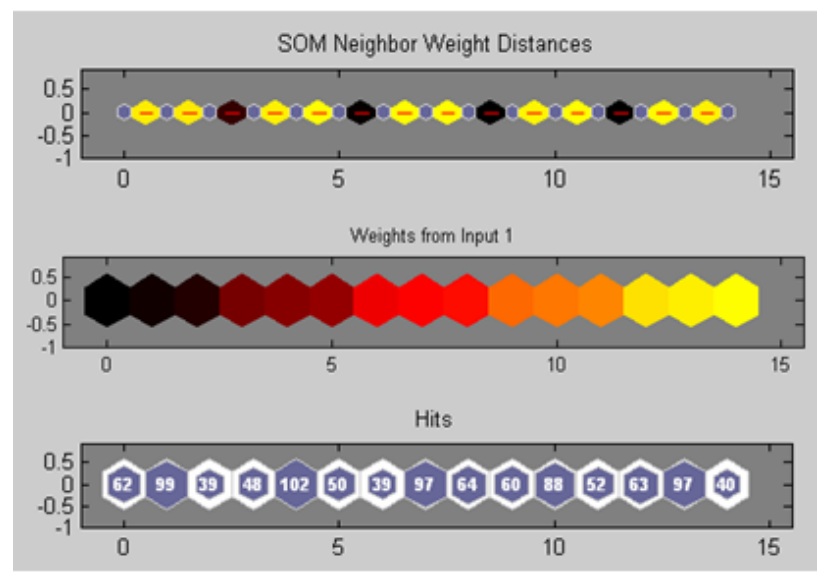

Fig. 10. Training results of self-organizing map.

The above two artificial neural network models constructs an intelligent measurement system which is capable of predicting the product quality and indentifying corresponding quality class for any given test sample. This method can also strongly help designers and other downstream parties conduct simulation based experiments to study the impact of designed settings or real settings on quality before real application. By comparing the values of the quality index generated on various simulated settings, the optimal setting could be found and applied before design regulation releases or before production starts.

\section{Knowledge for design and compliance improvement}

More data mining works, like correlation, distribution and comparison studies, can be conducted based on the above data, which help generate knowledge bases for optimizing quality assurance for both designers and other downstream parties.

\section{1) Correlation study}

For determining the quality pattern, a correlation study of the impact factors and quality change will reveal the critical impact factors that have bigger impact on quality. In probability theory and statistics, correlation (often measured as a correlation coefficient) indicates the strength and direction of a linear relationship between two random variables. A number of different coefficients are used for different situations. The best known is the Pearson productmoment correlation coefficient, which is obtained by dividing the covariance of the two variables by the product of their standard deviations, as shown below.

$$
\operatorname{Corr}(X, Y)=\rho_{X, Y}=\frac{\operatorname{cov}(X, Y)}{\sigma_{X} \sigma_{Y}}=\frac{E\left(\left(X-\mu_{X}\right)\left(\boldsymbol{Y}-\mu_{Y}\right)\right)}{\sigma_{X} \sigma_{Y}}
$$

where $\operatorname{Corr}(\boldsymbol{X}, \boldsymbol{Y})$ or $\rho_{X, Y}$ is correlation between two random variables $X$ and $Y, \operatorname{cov}(\boldsymbol{X}, \boldsymbol{Y})$ means covariance between $\mathrm{X}$ and $\mathrm{Y}, \sigma_{\boldsymbol{X}}, \sigma_{\boldsymbol{Y}}$ are standard deviations, $\boldsymbol{u}_{\boldsymbol{X}}, \boldsymbol{u}_{\boldsymbol{Y}}$ are expected values, and $\boldsymbol{E}$ is the expected value operator.

The correlation is 1 in the case of an increasing linear relationship, -1 in the case of a decreasing linear relationship, and some value in between these two values in all other cases, indicating the degree of linear dependence between the variables. The closer the coefficient is to either -1 or 1 , the stronger the correlation between the variables (see Table 4).

TABLE III. INTERPRETATION OF THE SIZE OF A CORRELATION.

\begin{tabular}{ccc}
\hline Correlation & Negative & Positive \\
\hline Small & -0.3 to -0.1 & 0.1 to 0.3 \\
Medium & -0.5 to -0.3 & 0.3 to 0.5 \\
Large & -1.0 to -0.5 & 0.5 to 1.0 \\
\hline
\end{tabular}

All 1000 samples were selected for this correlation study. The correlations of each impact factor and quality index are plotted in a Radar chart (see top left chart of Figure 11). The results reveal that seven factors have stronger correlation with quality for the samples studied. Three are raw material factors, namely, seating out of alignment (Rsa), mounting pressure $(R m p)$, and Lubricant $(R l u)$; two are machine status factors, namely, Health index (Ahi), Precision (Apr), and Balance $(A b a)$; and two are manufacturing environment factors: Humidity $(E h u)$, and Temperature (Ete).

\section{2) Distribution study}

As the quality index can be traced back to the original collected impact factors data, for all bad quality indices, a further distribution analysis of the parameter settings of each critical factor can be conducted to illustrate that what range it is most in, which is very important information to posses for impact factor design.

The distribution of all settings of the impact factor Rsa in very bad quality class (i.e. Class 5) is shown on top right chart in Figure 11. The range of settings is one type of feature of the root causes of bad quality, and should be avoided in design.

\section{3) Comparison study}

For such parties as material, manufacturing, and delivery, they can simulate their real setting and predict the quality index before production using the proposed expert system. If the predicted quality is bad or very bad, then the system can do an analysis to check the difference between the impact factors settings of the bad quality predicted and those settings in a very good quality pattern.

The bottom chart on Figure 11 presents an example of comparison, where the mean and range of each impact factor setting in very good quality pattern are compared with the real setting of each factor in test sample 2, which generated a very bad quality. The differences will feedback to instruct people how to adjust their settings to improve the compliance with design.

\section{J. System level quality assurance}

The procedures introduced in Section 3.3 to 3.6 are applied to all critical components. After all component level quality improvements have been completed, the system level quality will be improved as well. Like the impact factors in the component level quality assurance model, the impact factors for the system level quality assurance model are the factors influencing the quality of the wind turbines received by customers. The system level quality index is defined by customer requirements. A system level quality assurance 
model can be constructed in the same way as that at the component level. By using the Feedforward neural network and SOM methods, the quality index of a wind turbine system can then be predicted and the pattern of quality index can also be identified. Useful knowledge can be obtained from data mining. By continuously measuring the quality index, classifying its pattern and redefining the impact factors' settings, quality can be improved at both the system level and the component level until the quality index reaches the predetermined threshold.

\section{CONCLUSION AND FUTURE WORK}

The importance of performing quality assurance throughout an entire supply chain has been gradually gaining recognition by the decision makers in industry. In this study, the frameworks of collaborative quality assurance in a supply chain, including management model, technical model, and database model, were proposed so as to ensure high quality of products. In the wind turbine case study presented, bearings have been identified as an important component of wind turbines, and taken as an example to demonstrate the proposed component level quality assurance approach.

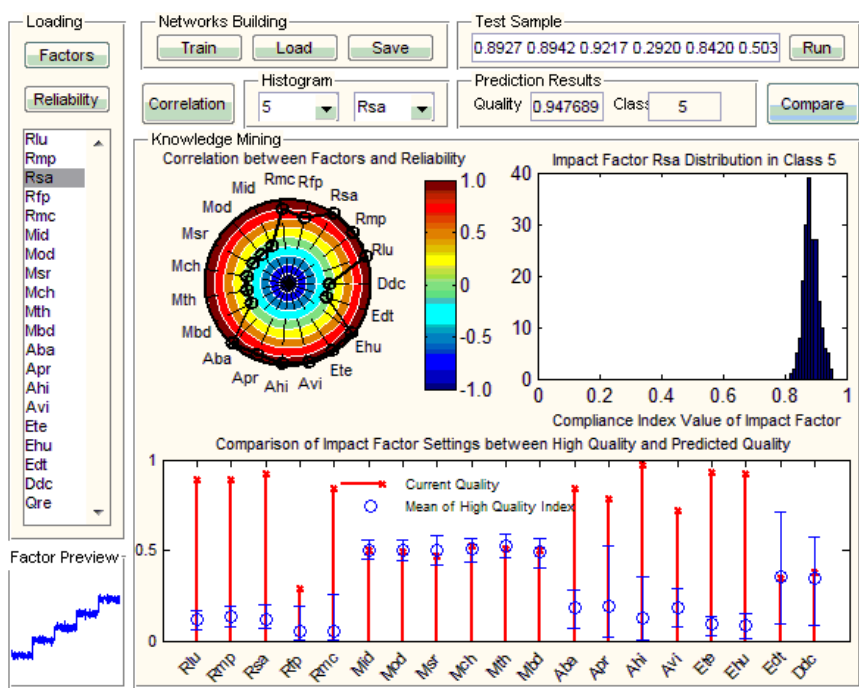

Fig. 11. Knowledge mining results in quality assurance expert system

The component quality assurance model acted as an intelligent measurement system, to predict quality and recognize the quality pattern given a set of quality impact factors. With these promising functions, designers can test whether the design settings will result in a good or bad quality product before releasing the design to downstream parties. Downstream parties, such as those responsible for manufacturing, can simulate different real settings to determine which may generate the best quality before production. More knowledge is mined based on the results through statistical analysis, such as correlation, distribution, and comparison, and feedback to designers and downstream parties for optimizing settings and tighter tolerance. With this intelligent quality assurance model, potential losses from bad design settings or incorrect real settings will be saved, and product quality will be ensured.

\section{REFERENCES}

[1] R. Early, A Guide to Quality Management Systems for the Food Industry. Blackie Academic and Professional, 1995, London.

[2] R. N. Baines and P. Ryan, Global trends in quality assurance. Trade Partners UK and Ministry of Agriculture "Modern Food Chain" Seminar, 2002, Kuala Lumpur.

[3] J. C. Turner and W. P. Davies, The modern food chain: profiting from effective integration. Trade Partners UK and Ministry of Agriculture 'Modern Food Chain' Seminar, 2002, Kuala Lumpur.

[4] L. Manning, R. N. Baines and S. A. Chadd, Quality assurance models in the food supply chain. British Food Journal, 2006, 108(2), 91-104.

[5] D. H. Stamatis, Failure Mode and Effect Analysis: FMEA from Theory to Execution, 2003, Hardcover.

[6] W. A. Shewhart, Economic control of quality of manufactured product. 1931, (D. Van Nostrand Company: New York).

[7] W. A. Shewhart, Statistical Method from the Viewpoint of Quality Control. 1939, (Dover: New York).

[8] G. Taguchi, Quality engineering (Taguchi methods) for the development of electronic circuit technology. IEEE Transactions on Reliability (IEEE Reliability Society) 1995, 44 (2), 225-229.

[9] D. C. Montgomery, Introduction to Statistical Quality Control (6th edition), John Wiley \& Sons, 2008, New York.

[10] J. Behnamian, S.M.T. Ghomi, Fatemi and M. Zandieh, Development of a hybrid metaheuristic to minimize earliness and tardiness in a hybrid flowshop with sequence-dependent setup times. International Journal of Production Research, 2009, 1-24, iFirst.

[11] X. Shi, P. Schillings, and D. Boyd, Applying artificial neural networks and virtual experimental design to quality improvement of two industrial processes. International Journal of Production Research, 2004, 42(1), 101-118.

[12] J.A. Andrawus, J. Watson, and M. Kishk, Wind Turbine Maintenance Optimisation: principles of quantitative maintenance optimization, Wind Engineering, 2007, 31 (2), 101-110.

[13] Winstats Newsletter, Section: Wind turbine data summary tables, 17, 2004.

[14] H. Braam, and L. Rademakers, Models to analyse operation and maintenance aspects of offshore wind farms. ECN Report, 2004.

[15] P. Tavner, G. V. Bussel, and F. Spinato, Machine and converter reliabilities in wind turbines. PEMD 06, 2006, 127-130.

[16] P. Tavner, J. Xiang and F. Spinato, Reliability analysis for wind turbines. Wind Energy, 2007, 10, 1-18.

[17] D. McMillan, and G. W. Ault, Specification of Reliability Benchmarks for Offshore Wind Farms. Proc. European Safety and Reliability, 2008.

[18] D. McMillan, and G. W. Ault, Condition monitoring benefit for onshore wind turbines: sensitivity to operational parameters. IET Renewable Power Generation, 2008, 2, 60-72. 